\title{
Microsymposia
}

MS.44.2

Acta Cryst. (2011) A67, C106

\section{Measuring lattice distortions from HR(S)TEM images}

Martin Hÿtch, Axel Lubk, Christophe Gatel, Etienne Snoeck CEMES-CNRS and Université de Toulouse, Toulouse (France). Email: hytch@cemes.fr

High-resolution transmission electron microscopy (HRTEM) and high-angle annular dark-field imaging in scanning transmission mode (HAADF-STEM) provide a wealth of information about atomic configurations and lattice distortions at the nanoscale. In principle, these HR(S)TEM images can be analysed using similar quantitative techniques but each have their own specificities. Here, we present analysis using two techniques: geometric phase analysis [1] and modelbased analysis of image contrast [2]; and gives results for two types of images: HRTEM and HAADF-STEM.

Geometric phase analysis (GPA) is best suited for the determination of the distortion of the crystalline lattice over large fields of view but at nanometre resolution, rather than atomic. Model-based analysis relies on fitting the contrast of individual atomic columns and is therefore more suitable to measuring displacements at the very highest resolution. Indeed, the displacements of atomics columns within the unit cells can be monitored across defects and interfaces with this technique, something impossible for GPA. In principle, model-based analysis is better adapted to the analysis of HAADF-STEM images than HRTEM whilst GPA is equally applicable to both.

When comparing TEM and STEM modes, TEM still gives the larger field of view, though recent progress in scan control is reducing the difference. Nevertheless, HRSTEM imaging still suffers from scan distortions which hamper the measurement of atomic displacements. HAADF-STEM provides images of thicker crystal which are in general more easily interpretable than HRTEM. Aberration correction has benefited both techniques, producing images with superior signalto-noise and hence results with higher precision, as the examples will show.

[1] M.J. Hÿtch, E. Snoeck, R. Kilaas, Ultramicroscopy 1998, 74, 131-146. [2] A.J. den Dekker, S. Van Aert, A. van den Bos, D.V. Dyck, Ultramicroscopy, 2005, 104, 83-106.

Keywords: transmission_electron_microscopy, image_analysis, strain

\section{MS.44.3}

Acta Cryst. (2011) A67, C106

\begin{abstract}
Structures of catalytically important nano-structured materials revealed by TEM

Hu Young Jeong, ${ }^{a}$ Kyoungmin Jung, ${ }^{a}$ Yusuke Yamauchi, ${ }^{b}$ Jun-Mo Yang, ${ }^{\mathrm{c}}$ Jeong Young Park, ${ }^{\mathrm{a}}$ Osamu Terasaki, ${ }^{\mathrm{a}, \mathrm{d}}{ }^{a}$ Graduate School of EEWS, KAIST, Daejeon, (Korea). ${ }^{b}$ World Premier International (WPI) Research Center, National Institute for Materials Science (NIMS), Tsukuba, (Japan). 'Measurement \& Analysis Team, National Nanofab Center, Daejeon, (Korea). ${ }^{\mathrm{d} D}$ Department of Materials and Environmental Chemistry, Stockholm University, Stockholm, (Sweden). E-mail: terasaki@kaist.ac.kr
\end{abstract}

As the size of metal particles approach to nanometer range, they have considerable scientific and technological significance in the catalytic field [1]. Despite the wealth of experimental and theoretical data, it remains unclear what kind of structure they have. The most straightforward one is transmission electron microscopy (TEM) to identify the structure of metal nanoparticles [2]. In particular, the spherical aberration (Cs) corrected scanning TEM is extensively used for extracting atomic and chemical information through a quantitative high-angle annular dark-field (HAADF) image which is remarkably senstive to the atomic number $(\mathrm{Z})[3]$ and number of atoms along the electron beam [4]. Here we show some examples for atomic-level characterization of metal nanoparticle.

Three dimensionally connected mesoporous Pt nanonetworks were synthesized within the mesopores of silica with Ia-3d symmetry and their structure was characterized. The Pt nanonetwork was formed with nearly single fcc crystalline nature and there was no specific orientation relationship between the Pt nanonetwork and original silica mesoporous crystal. Interestingly an envelope surface of the Pt nanonetwork showed rhombic dodecahedron.

TEM structural characterisation was also performed for ultrafine $\mathrm{Ru}$ and $\mathrm{Rh}$ nanoparticles synthesized by a polyol reduction method. We used free-standing graphene sheets as supporting film to obtain a clear image of sub $3 \mathrm{~nm}$ nanocrystals. By reducing background signal, we could obtain clear lattice-resolved image of nanoparticles. Using high resolution TEM (HRTEM) and Z-contrast STEM images, we could determine the shape and structure of each metal nanoparticles. $\mathrm{Ru}$ nanoparticles had a multiply twinned polyhedral (a face centered cubic structure) and triangular nanoprismatic shape (a hexagonal close packed structure). On the other hand, Rh nanoparticles showed a similar morphology, but only having a face centered cubic structure.

[1] T.S. Ahmadi, Z.L. Wang, T.C. Green, A. Henglein, M.A. El-sayed, Science 1996, 272, 1924-1925. [2] Z.L. Wang J. Phys. Chem B 2000, 104, 1153-1175. [3] V. Ortalan, A. Uzun, B.C. Gates, N.D. Browning, Nature Nanotech. 2010, 5 , 843-847. [4] Y. Liu, CJ Jia, J. Yamasaki, O. Terasaki, F. Schueth, Angew. Chem. Int. Ed. 2010, 49, 5771-5775.

\section{Keywords: nanocrystal, TEM, Structure}

\section{MS.44.4}

Acta Cryst. (2011) A67, C106-C107

Imaging a sub-Ångström electron beam after scattering in a crystal

J. Etheridge, ${ }^{\mathrm{a}, \mathrm{b}} \quad$ S. Lazar, ${ }^{\mathrm{d}} \mathrm{C}$. Dwyer, ${ }^{\mathrm{a}, \mathrm{b}, \mathrm{c}} \mathrm{G} . \mathrm{A}$. Botton, ${ }^{\mathrm{e}} \quad{ }^{a}$ Departament of Materials Engineering, ${ }^{b}$ Monash Centre for Electron Microscopy, ${ }^{c} A R C$ Centre of Excellence for Design in Light Metals, ${ }^{a, b, c}$ Monash University, Victoria, (Australia). ${ }^{d}$ FEI Electron Optics, $5600 \mathrm{KA}$ Eindhoven, (The Netherlands). ${ }^{e}$ Canadian Centre for Electron Microscopy and Department of Materials Science and Engineering, McMaster University, Hamilton, Ontario, (Canada). E-mail: joanne. etheridge@monash.edu

The ability to generate electron probes in transmission electron microscopes (TEM) with a diameter less than one Ångström has opened the possibility of obtaining information about the position, type and bonding of individual atoms selected within a specimen. However, to extract this information, it is critical to understand how a sub-Ångström electron probe scatters within a crystal. Calculations have shown [14] that the electron probe rapidly disperses from its impact point onto and between adjacent atomic columns, so that the spatial origin of the final scattered signal is not easily attributable to specific atoms within the specimen. Here we describe a method for imaging the distribution of a sub-Ångström electron probe in real space after scattering from a crystal, using a double-aberration corrected TEM. We image the scattered electron intensity in real space with sub-Ångström resolution for a sequence of 10 incident probe positions located at $0.3 \AA$ intervals within a unit cell of $\mathrm{Au}$ [5]. These images reveal the acute sensitivity of the electron distribution to these tiny shifts in the probe position and to the local site symmetry of the incident probe. We also image the intensity distribution after excitation of a plasmon for different incident probe positions within a unit cell of $\mathrm{LaB}_{6}$, which are again 


\section{Microsymposia}

acutely sensitive to local site symmetry. Furthermore, these inelastic images illustrate the complex nature of the scattering from the boron octahedra. The implications for extracting information about individual atoms within a specimen using conventional quantitative STEM and using direct images of the scattered electron probe will be discussed.

[1] K. Ishizuka, J. Electron Microsc. 2001, 50, 291-305. [2] C. Dwyer, J. Etheridge, Ultramicroscopy 2003, 96, 343-360. [3] C.J. Rossouw, L.J. Allen, S.D. Findlay, M.P. Oxley, Ultramicroscopy 2003, 96, 299-312. [4] P. Voyles, Grazul, D.A. Muller Ultramicroscopy 2003 96, 251. [5] J. Etheridge, S. Lazar, C. Dwyer, G.A. Botton, Phys Rev. Lett 2011, 106, 160802.

Keywords: scanning transmission electron microscopy, electron scattering, double-aberration correction.

\section{MS.44.5}

Acta Cryst. (2011) A67, C107

\begin{abstract}
QFocus: Structure reconstruction from focal series of hrtem images

Xiaodong Zou, ${ }^{\mathrm{a}, \mathrm{b}}$ Wei Wan, ${ }^{\mathrm{a}, \mathrm{b}}$ Sven Hovmöller, ${ }^{\mathrm{a}}$ 'Department of Materials and Environmental Chemistry, Stockholm University, SE10691 Stockholm, (Sweden). ${ }^{\mathrm{b}}$ Berzelii Centre EXSELENT on Porous Materials, Stockholm University, SE-106 91 Stockholm, (Sweden). Email: xiaodong.zou@mmk.su.se
\end{abstract}

High-resolution transmission electron microscopy (HRTEM) images are usually not directly interpretable in terms of crystal structure due to various aberrations in the microscope lenses. Although significant progress has been made recently in the development of hardware aberration correctors, especially spherical-aberration correctors [1], the majority of transmission electron microscopes in use today are traditional ones and structure reconstruction by software methods still serves as a cost-effective alternative. Representative software methods include exit wave reconstruction using throughfocus image series [2] and contrast transfer function (CTF) correction methods using single images or image series [3]. Here we present a CTF-correction based structure reconstruction method, using throughfocus series of HRTEM images taken with a fixed focus step, in which all images in the series are corrected for CTFs and combined into a structure image. To determine the starting defocus of the series, trial defocus values in a large range are tested and CTF corrections are made to all images. The similarity of the phases of the Fourier transform between the corrected images is used as the criterion to judge whether a trial defocus value is close to the true one. The two-fold astigmatism is determined by dividing Fourier transforms into sectors and determining the defocus along different directions. As crystallographic phases are used in defocus and astigmatism determination, no amorphous areas are required in the images. On the other hand, since CTF correction is done for all pixels within the resolution limit in reciprocal space, this method works for both perfect and defect crystals.

The method was applied on a 20-image focal series of $\mathrm{Ca}_{0.28} \mathrm{Ba}_{0.72} \mathrm{Nb}_{2} \mathrm{O}_{6} \quad(P 4 b m, \quad a=12.43 \AA, \quad c=3.96 \AA)$. The experimental images were collected with a focus step of $-26.6 \AA$ on a JEOL-2100F transmission electron microscope equipped with field emission gun. The starting defocus of the series is determined to be $-260 \AA$ and the two-fold astigmatism is $33 \AA$ with an azimuth angle of $13^{\circ}$ clockwise with respect to the $x$-axis of the images. The reconstructed image is shown in the figure below, in which atoms appear as black dots. Not only metals but also atomic columns as light as oxygen can be seen.

The present method has the advantages of automatic defocus and two-fold astigmatism determination without amorphous areas, less CTF crossover problems and improved signal-to-noise ratio as compared to CTF correction methods using single images. The method has been implemented in a user-friendly program, QFocus, which we hope may help non-TEM experts to use HRTEM images for solving their structure problems.

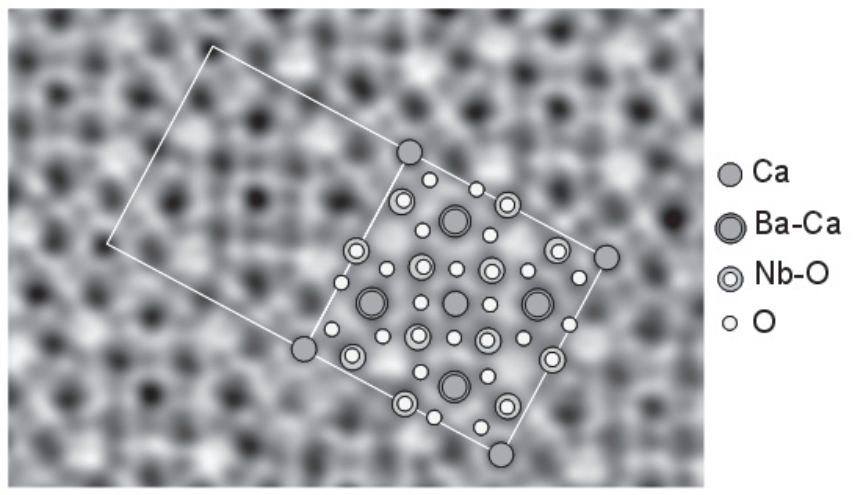

[1] M. Haider, H. Rose, S. Uhlemann, E. Schwan, B. Kabius, K. Urban, Nature 1998, 392, 768-769. [2] W.M.J. Coene, A. Thust, M. Op de Beeck, D. Van Dyck, Ultramicroscopy 1996, 64, 109-135. [3] X.D. Zou, M. Sundberg, M. Larine, S. Hovmöller, Ultramicroscopy 1996, 62, 103-121.

Keywords: HRTEM, structure reconstruction, electron crystallography

\section{MS.45.1}

Acta Cryst. (2011) A67, C107-C108

"Making the molecular movie": First frames...coming features

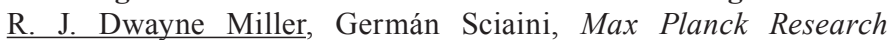
Department for Structural Dynamics, Department of Physics, University of Hamburg and Centre for Free Electron Laser Science, DESY, Notkestrasse 85, Hamburg, (Germany). Department of Chemistry and Physics, University of Toronto, 80 St. George, Toronto (Canada).E-mail: dmiller@1phys.chem.utoronto.ca.

One of the great dream experiments in Science is to watch atomic motions as they occur during structural changes. In the fields of physics, chemistry and biology, this prospect provides a direct observation of the very essence of chemistry and the central unifying concept of transition states in structural transitions. From a physics perspective, this capability would enable the observation of rarified states of matter at an atomic level of inspection, with similar important consequences for understanding nonequilibrium dynamics and collective phenomena. This experiment has been referred to as "making the molecular movie". Due to the extraordinary requirements for simultaneous spatial and temporal resolution, it was thought to be an impossible quest and discussed in the context of the purest form of a gedanken experiment. Recent developments in femtosecond electron guns with sufficient brightness to even execute single-shot structural determinations have made this experiment become a reality [1]. Previously thought intractable problems in attaining sufficient brightness and spatial resolution, with respect to the inherent electron-electron repulsion or space charge broadening, have been solved. With this new level of acuity in observing structural dynamics, there have been many surprises and this will be an underlying theme. Several movies depicting atomic motions during passage through structural transitions relevant to condensed phase dynamics will be shown [2], [3], [4]. The primitive origin of molecular cooperativity has also been discovered in recent studies of molecular crystals. These new developments will be discussed in the context of developing the necessary technology to directly observe the structure-function correlation in biomolecules - the fundamental molecular basis of biological systems. The future is even brighter with the advent of a new concept in relativistic electron guns that will open up direct observation of atomic motions in solution and 